\title{
The strong Pc5 geomagnetic pulsations in the initial phase of the great magnetic storm of March 24, 1991
}

\author{
J. J. Schott ${ }^{1}$, N. G. Kleimenova ${ }^{2}$, J. Bitterly ${ }^{1}$, and O. V. Kozyreva ${ }^{2}$ \\ ${ }^{1}$ Ecole et Observatoire des Sciences de la Terre (EOST), 67084 Strasbourg, France \\ ${ }^{2}$ Institute of Physics of the Earth, 123810 Moscow, Russia
}

(Received November 5, 1996; Revised November 10, 1997; Accepted January 6, 1998)

\begin{abstract}
Pc5 pulsations which were excited during the great magnetic storm of March 24, 1991 are analysed using the data from three French observatories located in the southern Indian Ocean at the subauroral and middle latitudes. The unusual strong Pc5 pulsations observed display features depending on the interval of occurrence: a quasi-noisy regime in the frequency range of $2.5-3.2 \mathrm{mHz}$ characterizes the 04-10 UT interval, whereas the oscillations are nearly monochromatic with spectral maximum around $1.8-1.9 \mathrm{mHz}$ in the 10-14 UT interval. The sharp change occurring at 10.17 UT could be associated to a sharp change in the magnetosphere structure or dynamics.
\end{abstract}

\section{Introduction}

The study of morphology and nature of the Pc5 ( $f \sim 2-6$ $\mathrm{mHz}$ ) geomagnetic pulsations observed on the ground level have been the subject of many papers. A series of recent publications reported the observation of power enhancement of wave spectra at discrete frequencies (near $1.3 \mathrm{mHz}, 1.7-$ $1.9 \mathrm{mHz}, 2.3-2.6 \mathrm{mHz}$ and $3.2-3.5 \mathrm{mHz}$ ), which corresponds to field line resonance (Chen and Hasegawa, 1974; Southwood, 1974) apparently driven by magnetosphere cavity or waveguide modes (Ruohoniemi et al., 1991; Samson et al., 1991; Harrold and Samson, 1992; Samson and Harrold, 1992; Walker et al., 1992; Ziesolleck and McDiarmid, 1994).

Field line resonances can be excited by several relatively broad sources such as Kelvin-Helmoltz instabilities, transient day reconnection, step variations of the solar wind dynamic pressure (Dunlop et al. (1994) and references therein). It is well established that, on the Earth's surface, Pc5 waves propagate with small azimuthal wave numbers to the West in the morning side, and to the East in the afternoon.

Geomagnetic pulsations Pc5 are typical phenomena at high latitudes during recovery phases of magnetic storms and substorms (see for example, Engebretson et al., 1983). The maximum of the Pc5 amplitudes are usually observed in a relatively narrow geomagnetic latitude range (typically $68^{\circ}-72^{\circ}$ ). Their region of occurrence coincides with the auroral oval. The amplitude of Pc5 sharply decreases in function of their distance from the generation region. Pc5 pulsations with amplitudes larger than $10 \mathrm{nT}$ are seldom observed at latitudes lower than $60^{\circ}$.

It is well known that the development of strong magnetic storms leads to a significant reconstruction of large scale structures of the magnetosphere. For instance, auroral zones

Copy right (C) The Society of Geomagnetism and Earth, Planetary and Space Sciences (SGEPSS); The Seismological Society of Japan; The Volcanological Society of Japan; The Geodetic Society of Japan; The Japanese Society for Planetary Sciences. can extend to subauroral and even middle latitude areas. In such extreme situations, Pc5 pulsations can be observed at unusually low latitudes and, in such instances, the analysis of geomagnetic observations at subauroral and middle latitudes provides useful information. An exceptional strong magnetic storm happened on March 24, 1991. According to Bell et al. (1997), it belongs to the "super storm" class for it is characterized by an exceptionally large geomagnetic sudden commencement (SSC) occurring at 03.41 UT (Araki et al., 1997), Dst $=-298 \mathrm{nT}$ (24 UT) and $A E$ as large as $\sim 4000$ nT at 04-05 UT. The Dst index decreased in the 04-10 UT interval from $+63 \mathrm{nT}$ to $-98 \mathrm{nT}$ and increased in the 10-14 UT interval from $-98 \mathrm{nT}$ to $-57 \mathrm{nT}$.

The global burst of long period ( $T \sim 10 \mathrm{~min}$ ) geomagnetic pulsations at 12-14 UT was observed by Fujitani et al. (1993), Liu et al. (1993), Reddy et al. (1994). Fujitani et al. (1993) using multi-stations data from several meridian chains demonstrated the global character of the 12-14 UT Pc5's, having the strongest amplitudes in the afternoon sectors and periods independent on latitudes. The properties of these pulsations are different from those of typical Pc5 caused by field line resonance. Liu et al. (1993), using CW-HF Doppler frequency sounding system and fluxgate magnetometers at the station of Taiwan, showed beautiful sinusoidal oscillations with phase differences of $15^{\circ}-77^{\circ}$. Reddy et al. (1994) found similar oscillations with coherent backscatter radar and magnetometer records. These observations were interpreted in terms of compressional cavity mode resonance in the inner magnetosphere and associated ionospheric electrical field penetrating from high latitude toward the magnetic equator.

Occurring before the global burst of Pc5 pulsations with periods of about $10 \mathrm{~min}$, discussed by Fujitani et al. (1993), Liu et al. (1993), and Reddy et al. (1994), we have observed another type of unusual Pc5's with shorter periods. The aim of our paper is to study these strong Pc5 pulsations occurring in the 06-10 UT time interval during these untypical conditions, i.e. in the initial phase of the magnetic storm. 


\section{Data and Results}

In this paper, the morphological peculiarities of Pc5 pulsations are analysed using digital one minute sampling data obtained from the French observatories located in the Southern Indian Ocean. The coordinates of these stations are listed in Table 1.

The SSC occurring at 03.41 UT led to the development of a large magnetic substorm. Even in the late local morning at
$L=3.6$ (PAF) the amplitude of the $H$-component reached 1800 $\mathrm{nT}$ (in comparison, the maximum amplitude was $2300 \mathrm{nT}$ at auroral observatory Sodankyla (SOD), located in the northern hemisphere $\left(\Phi^{\prime}=63.9^{\circ}, \Lambda^{\prime}=109^{\circ}, L=5.1\right)$. The electrojet very quickly shifted towards the equator, whereas the current intensity sharply changed along meridians, what can be noticed by comparison of the records from CZT and AMS: at AMS located at a lower latitude than CZT, but $30^{\circ}$

Table 1. List of the observatories used in this study.

\begin{tabular}{|c|c|c|c|c|c|c|c|}
\hline \multirow[t]{2}{*}{ Station name } & \multirow[t]{2}{*}{ Abb. code } & \multicolumn{2}{|c|}{ Geographic } & \multicolumn{2}{|c|}{ Corrected } & \multirow[t]{2}{*}{$L$} & \multirow[t]{2}{*}{ MLT } \\
\hline & & lat. & long. E & lat. & long. & & \\
\hline Kerguelen (Port aux-Français) & PAF & -49.35 & 70.20 & -58.25 & 121.55 & 3.61 & $\mathrm{UT}+3.4$ \\
\hline Crozet (Port Alfred) & $\mathrm{CZT}$ & -46.43 & 51.87 & -53.47 & 105.55 & 2.82 & $\mathrm{UT}+2.4$ \\
\hline Amsterdam (Martin de Vivies) & AMS & -37.83 & 77.57 & -48.51 & 137.88 & 2.28 & $\mathrm{UT}+4.5$ \\
\hline
\end{tabular}

\section{PAF}
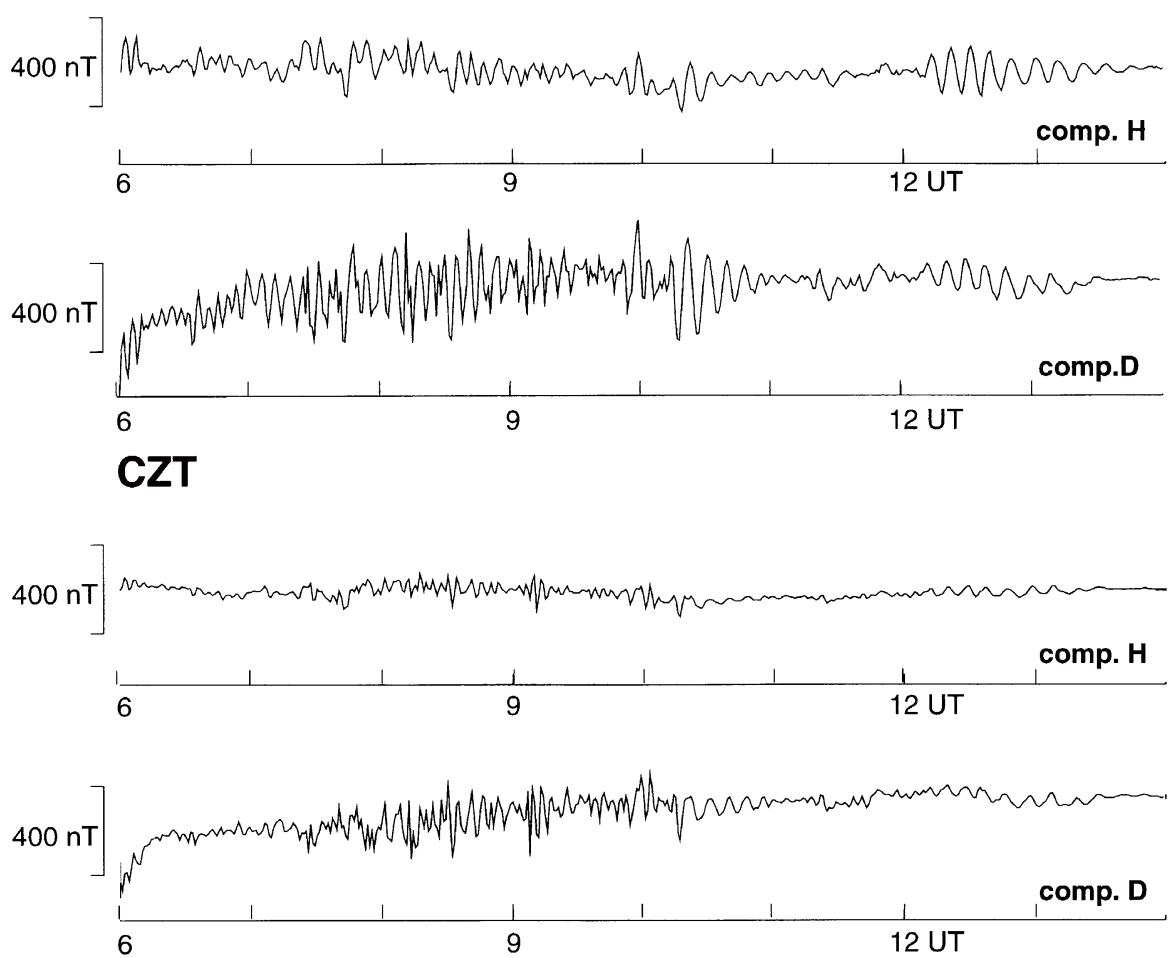

AMS


Fig. 1. $H$ and $D$ magnetograms in the interval 06-14 UT on March 24, 1991 for PAF, CZT and AMS. Note the change in the pulsation regime occurring at $10.17 \mathrm{UT}$. 
(a) $\mathrm{nT}^{2} / \mathrm{mHz}$
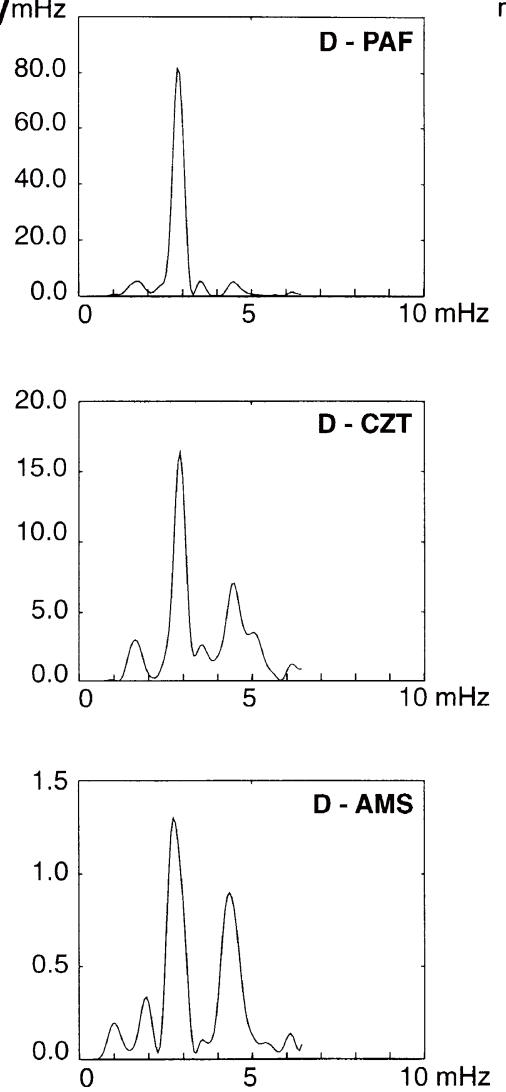
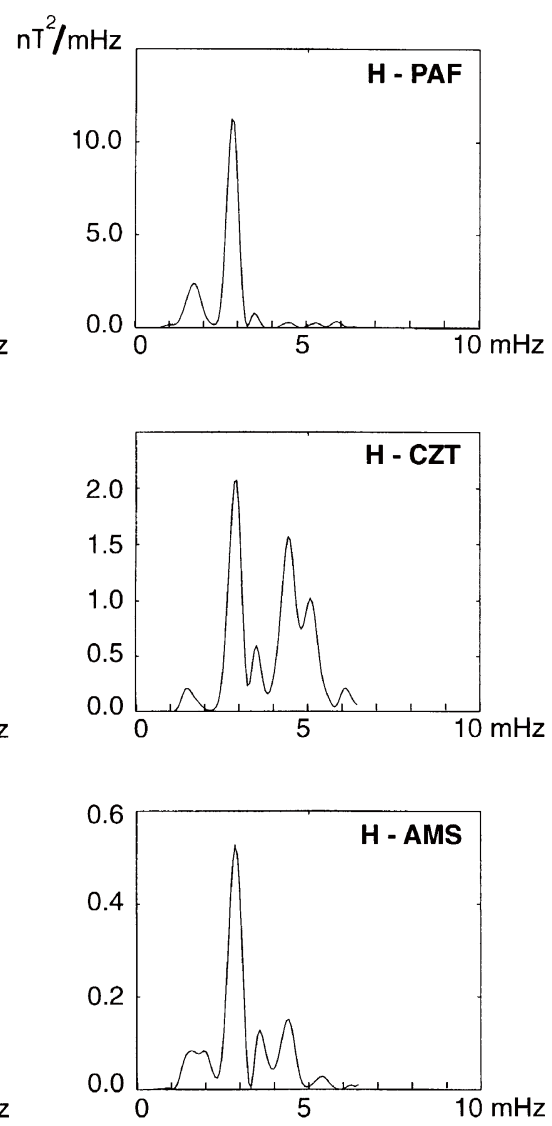

(b) $\mathrm{nT}^{2} / \mathrm{mHz}$
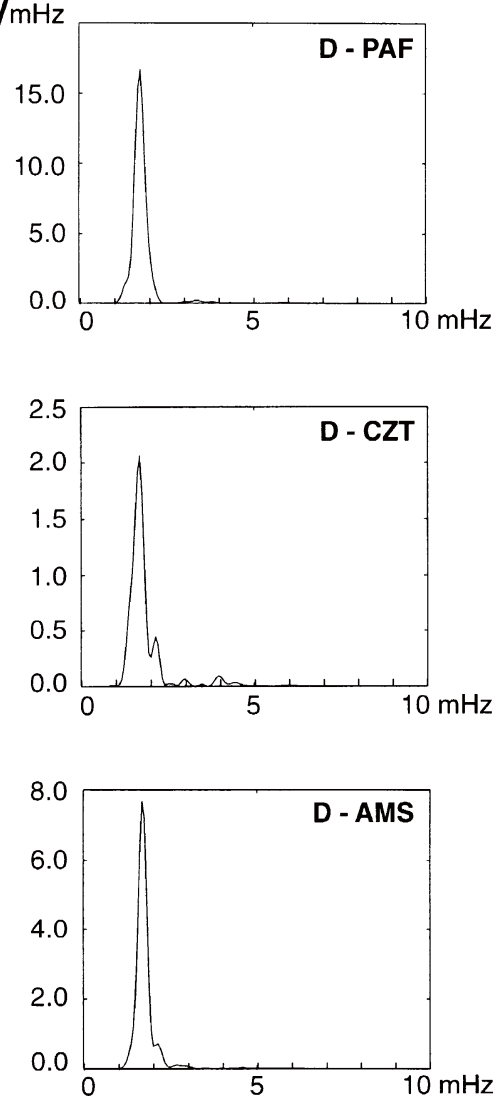
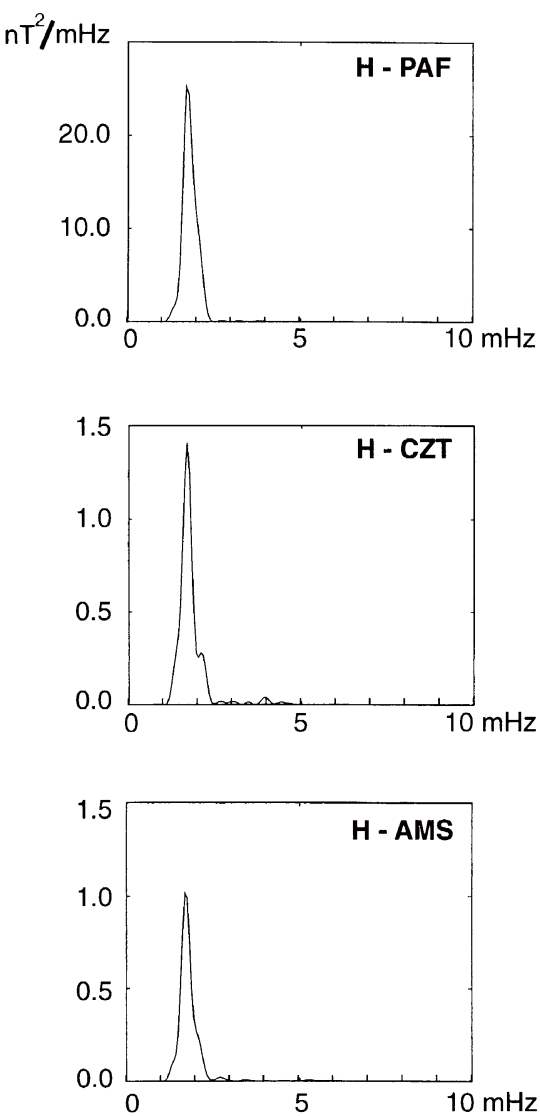

Fig. 2. $H$ and $D$ spectra of PAF, CZT and AMS computed for 08-09 UT (a) and 12-13 UT (b). Note the strong change in frequency content between the two selected intervals: the afternoon regime is nearly monochromatic. 
farther East, the substorm amplitude was larger than at CZT. This observation is consistent with the evolution of the $A E$ index which was as large as $\sim 4000 \mathrm{nT}$ at 04-05 UT and very rapidly decreased to $\sim 800 \mathrm{nT}$ at $07-08 \mathrm{UT}$.

Magnetic pulsations in the Pc5 range were observed in all three stations during the recovery phase of this substorm. Non-filtered pulsations data are presented in Fig. 1. The Pc5 observations can be subdivided into two intervals: 04-10 UT and 10-14 UT. The first Pc5 regime is characterized by quasi-noisy pulsations with amplitudes decreasing with latitudes (which can be clearly seen if we compare for instance the $H$-component values reached at PAF (150-160 nT) with those observed at CZT (60-70 nT) and at AMS (30-40 nT). The D-component amplitudes at all stations were twice larger than the $H$-component. The pulsations look like a separate packet structure occurring simultaneously at all latitudes, which means that either their sources are the same irregular impulses on the magnetopause or they are the result of the superposition of several simultaneous sources.

Spectral analysis (performed with the periodogram method using a Hamming window) shows that the main peak (both in $H$ and $D$ components) appears in the range $2.5-3.2 \mathrm{mHz}$ at all three stations (Fig. 2(a)). The detailed study of the late morning pulsations filtered in the frequency range 2.0-3.5 $\mathrm{mHz}$ (Fig. 3) shows clear phase delay between AMS and CZT $\left(30^{\circ}\right.$ longitude difference). We can infer that the waves propagated from noon (AMS) to morning (CZT) side of the magnetosphere (Fig. 3(a)), with a small azimuthal wave number $(m \sim 2-3)$. In the afternoon, the geomagnetic pulsations in the frequency range $1.5-2.0 \mathrm{mHz}$ (Fig. 3(b)) propagated in the opposite direction, from noon towards the evening side (i.e. from CZT to AMS). This observation supports the hypothesis of a subsolar source location (Dunlop et al., 1994).

In the 06-10 UT interval, Pc5 pulsations were observed at many stations, located in the local morning and near noon (Europe, Greenland). In contrast to typical morning Pc5's, the pulsations described above have similar spectra, characterized by the relatively narrow maximum of $2.5-3.2 \mathrm{mHz}$ independent on latitude (for example, from Godhavn, $\Phi^{\prime}=$ $77^{\circ}, 04-08 \mathrm{MLT}$ to Amsterdam, $\left.\Phi^{\prime}=48^{\circ}, 10-14 \mathrm{MLT}\right)$ with the maximum of amplitude $(\sim 500 \mathrm{nT})$ in the morning at Narssarssuaq $\left(\Phi^{\prime}=67^{\circ}\right)$. The lower latitude stations (CZT and AMS) spectra display a second maximum at $4-5 \mathrm{mHz}$, the largest peak being in the $D$-component at CZT.

A sharp change in pulsation regime occurs at 10.17 UT. The quasi-noisy character of pulsations suddenly changed into more regular, monochromatic oscillations. The spectral maximum shifts towards lower frequencies: $1.5-2.0 \mathrm{mHz}$ (Fig. 2(b)). In addition, at PAF and AMS, the polarization vector sense of rotation changed: counterclockwise before 10.17 UT and clockwise after 10.17 UT. The new regime was essentially monochromatic, displaying the most beautiful waves in the 12.00-13.30 UT interval. This burst of Pc5 pulsations was observed in a large longitudinal range, from geomagnetic meridian $80^{\circ}$ to meridian $210^{\circ}$, with the same period of about 9-10 min (Fujitani et al., 1993). As before, the period was independent of latitudes, and the amplitudes of the $H$-component of Pc5 decreased with decreasing latitudes (from $\sim 200 \mathrm{nT}$ at PAF to $\sim 40 \mathrm{nT}$ at CZT and AMS). The amplitudes of the first Pc5 regime $(2.5-3.2 \mathrm{mHz})$ were larger in the morning sectors of the Earth, but those of the second one $(1.5-2.0 \mathrm{mHz})$ larger in the afternoon.

In addition, a comparison of data at two pairs of nearly conjugate points, namely Kerguelen-Nurmiervi and CrozetBorok, showed in-phase variation of $H$-component and outof-phase variation of $D$-component. This supports the presence of the fundamental harmonic of standing waves.

To summarize, the main pecularities of the pulsations analysed above are 1) the latitude independency of their spectrum, in both regimes, quasi-noisy as well as nearly monochromatic, and 2) the sudden disappearance of the 2.5-3.2 mHz pulsations at 10.17 UT. This observation is at variance with the properties of the Pc5 pulsations observed during the great magnetic storm of March, 13, 1989, which was analysed by Bolshakova et al. (1995), using the Scandinavian meridian chain and PAF and CZT data. They showed that the main maximum in the late morning Pc5 spectrum shifted from $3.3 \mathrm{mHz}$ at PAF to $5.6 \mathrm{mHz}$ at $\mathrm{CZT}$, according to the field line resonance theory.

\section{Discussion}

The main pulsation period in the interval 6-10 UT was practically the same ( $T \sim 6 \mathrm{~min} ; f \sim 2.5-3.2 \mathrm{mHz})$ in all stations, independent on latitudes. The similarity of wave spectra at various latitudes and the nearly simultaneous appearance of separate wave packets allow us to conclude that they originated from a common source, for example, trigerred by a succession of impulses. Independence of the main spectral peak on latitudes over a considerably large latitude range, the narrow frequency range, and the amplitude of $D$-component larger than $H$-component at every latitude, do not fit with local field line resonances theory.

Several other assumptions may be imagined in order to predict the Pc5 pulsation generation in our case.

It could be the result of modulation (ringing) of a three dimensional field aligned current system (Lam and Rostoker, 1978; Lam, 1989), but this mechanism is very doubtful because the Pc5 pulsations were observed near local noon, after the end of the substorm at the given meridian.

Another possibility relies upon instabilities on the boundary between the low latitude boundary layers (LLBL) and the magnetopause, as it was proposed by Sonnerup (1980) and Engebretson et al. (1983). They observed similar Pc5 pulsations simultaneously in an extended latitude region both from Earth surface stations and on board the satellite Explorer 45 during the strong magnetic storm of August 5, 1972. The low latitude boundary layer was observed at $L \sim$ 4.5 during the storm time.

Kivelson et al. (1984) and Crowley et al. (1987) proposed a mechanism of wave generation connected with fast mode resonance of the entire magnetospheric cavity located between the magnetopause and the inner turning point or at least the sunward half of the cavity. Because of the radial gradient of Alfven speed, the turning point of waves could coincide with the plasmapause. According to analytical models of a global compression mode (for instance, Kivelson et al., 1984) the waves are reflected, on one side, on the sharp gradient of the magnetic field of the magnetopause and, on the other side, on the plasma density gradient near the 
plasmapause. The poloidal global mode is characterized by azimuthal electric field oscillations and radial motions of the plasma. In the meridian plan the frequency of poloidal fluctuations must be $L$-independent. The model cavity frequencies are sensitive to the magnetopause location. This model does not explain the stability of observed spectra both in 06-10 UT and 12-14 UT intervals with respect to geomagnetic conditions during such a strong magnetic storm.

The most relevant mechanism might be the model of wave guide modes proposed by Samson and Harrold (1992), Walker et al. (1992), Harrold and Samson (1992). The pulsations could be initiated by solar wind disturbances perturbing the magnetopause. As a result, waveguide modes would be excited in the cavity formed between the magnetopause and the turning point, where Alfven waves are reflected with phase speeds matching the disturbance speed. Each disturbance would emit a wave packet growing and decaying with a time scale controlled by the response of the magnetosphere state and the losses in the ionosphere. The azimuthal phase velocities of waveguide model should be comparable to the velocities of disturbances moving along the magnetopause near local dawn. The observed azimuthal wave number $m \sim 2-3$ for $\sim 2.5 \mathrm{mHz}$ oscillations corresponds to a velocity on the magnetopause of about $300 \mathrm{~km} / \mathrm{s}$ (Ruohoniemi et al.,1991), which is not contradictory with results of Samson and Harrold (1992).

In addition, it is interesting to mention that the spectra maxima $(\sim 2.7-2.8 \mathrm{mHz}$ in the $06-10 \mathrm{UT}, \sim 1.8-1.9 \mathrm{mHz}$ in the 12-14 UT) are consistent with some of the discrete peaks $(1.3 \mathrm{mHz}, 1.9 \mathrm{mHz}, 2.7 \mathrm{mHz}$ and $3.3 \mathrm{mHz}$ ) discussed by Samson et al. (1991), Walker et al. (1992), Samson and Harrold (1992), Ziesolleck and McDiarmid (1994).

A sharp change in the pulsation regime occurred simultaneously at all stations at 10.17 UT. Frequencies suddenly decreased from $2.5-3.5 \mathrm{mHz}$ to $1.5-2.0 \mathrm{mHz}$ with a maximum at $1.8 \mathrm{mHz}$, and the polarization became clockwise. At the same time, a short negative magnetic bay was observed on the nightside of the magnetosphere. Since Interplanetary Magnetic Field (IMF) data is not available, we can only assume that these events originated in a new compression of the magnetosphere by the solar wind. The increase displayed by the $D s t$ index supports this suggestion.

It is difficult to explain why the $2.5-3.2 \mathrm{mHz}$ pulsations suddenly turned off. A second difficulty arises from the existence of only one maximum in the spectra. According to Zhu and Kivelson (1994), compressional pulsations in the daytime magnetosphere are characterized by very low frequencies, are nearly monochromatic and extend over regions several Re large in the radial direction. This description matches the 12-14 UT Pc5's, which might be therefore identified as compressional waves caused by fluctuations of the solar wind dynamic pressure, as proposed by Fujitani et al. (1993).

Finally, the second maximum at $4-5 \mathrm{mHz}$ in the Pc5 spectra showed in Fig. 2 could result from Alfven field line resonance at a geomagnetic latitude of about $50^{\circ}$, provided by the second harmonic of the global mode. This peak is observed only in CZT and AMS, not in PAF, due to a strong wave dumping with increasing distance from the resonance region as it is predicted by theory (see, for instance, Chen and Hasegawa, 1974).

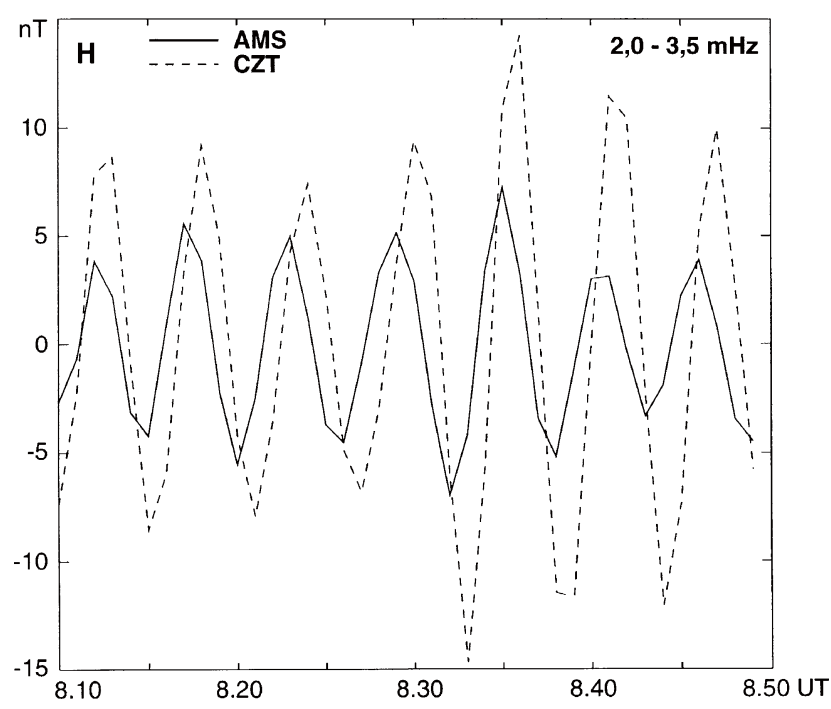

(a)

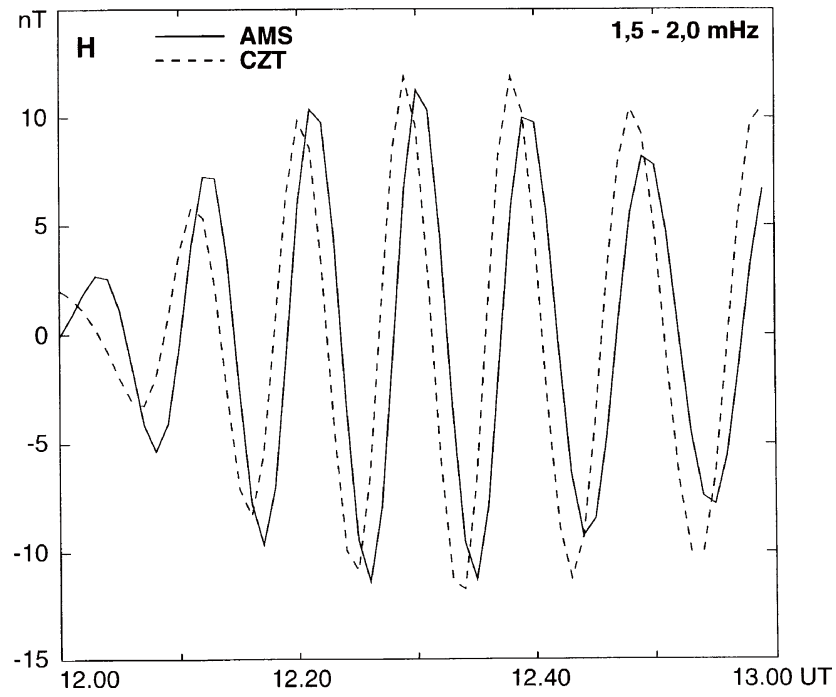

(b)

Fig. 3. Comparison between the band-filtered $H$-component variations at AMS and CZT: (a) 08.10-08.50 UT (2.0-3.5 mHz); (b) 12.00-13.00 UT $(1.5-2.0 \mathrm{mHz})$. Note the change of phase delay between the two intervals.

\section{Conclusion}

The intense Pc5 pulsations observed at unusual low latitudes during the great magnetic storm of March 24, 1991, cannot be classified as typical morning Pc5's connected with substorm developments and field line resonances. The pulsation spectrum in the 06-10 UT, as well as in the 12-14 UT interval, in contrast to typical Pc5, has only one latitude independent peak.

In addition, in contrast to ordinary Pc5's the reported pulsations were observed in the initial phase of this very strong storm. We propose that the source of the 06-10 UT pulsations is located near the subsolar point, in accordance with a previous assumption made by Dunlop et al. (1994). In our opinion, the most likely generation model refers to the magnetospheric MHD waveguide/cavity mode proposed by 
Samson et al. (1991), Walker et al. (1992), Samson et al. (1992). These rather unusual properties emphasize the dynamics of the magnetospheric processes triggered by this great magnetic storm, as seen from this restricted set of observatories.

Acknowledgments. We thank A. Lipton for her assistance with the English version. The drafts have been reworked by M. Blanck. Her help is gratefully acknowledged. This research was supported by Centre National de la Recherche Scientifique (CNRS), URA 323.

\section{References}

Araki, T., S. Fujitani, M. Emoto, K. Yumoto, K. Shiokawa, T. Ichinose, H. Luehr, D. Orr, D. K. Milling, H. Singer, G. Rostoker, S. Tsunomura, Y. Yamada, and C. F. Liu, Anomalous sudden commencement on March 24, 1991, J. Geophys. Res., 102, 14075-14087, 1997.

Bell, J. T., M. S. Gussenhoven, and E. G. Mullen, Super storms, J. Geophys. Res., 102, 14189-14198, 1997.

Bolshakova, O. V., O. K. Borovkova, Yu.F. Borovkov, N. G. Kleimenova, and J. Bitterly, Magnetic storm on March 13, 1989: The structure of extremely disturbed magnetosphere obtained from geomagnetic pulsations Pc5, Geomagn. Aeron., 34, 492-500, 1995 (English ed.).

Chen, L. and A. Hasegawa, A theory of long period magnetic pulsations. 1. Steady state excitation of field line resonance, J. Geophys. Res., 79 1024-1037, 1974.

Crowley, G., W. J. Hughes, and T. B. Jones, Observational evidence of cavity modes in the Earth's magnetosphere, J. Geophys. Res.,92, 1223312240, 1987.

Dunlop, I. S., F. W. Menk, H. J. Hansen, B. J. Fraser, and R. J. Moris, A multistation study of long period geomagnetic pulsations at cusp and boundary layers latitudes, J. Atmos. Terr. Phys., 56, 667-679, 1994.

Engebretson, M. J., L. J. Cahill, and D. J. Williams, Pulsations in magnetic field and ion flux observed at $L=4.5$ on August 5 1972, $J$ Geophys. Res., 88, 161-173, 1983.

Fujitani, S., T. Araki, K. Yumoto, K. Shiokawa, D. Orr, S. Tsunomura, Y. Yamada, H. Luhr, C. F. Lue, D. R. K. Rao, T. Kitamura, and K Hayashi, Pc5 pulsations appeared on March 24 1991, STEPGBRSC NEWS, 3, 15, 1993.

Harrold, B. G. and J. C. Samson, Standing ULF modes of the magnetosphere: a theory, Geophys. Res. Lett., 19, 1811-1814, 1992.
Kivelson, M. G., J. Etcho, and J. G. Trotignon, Global compressional oscillations of the terrestrial magnetosphere: the evidence and a model, J. Geophys. Res., 89, 9851-9856, 1984.

Lam, H. L., A possible classification of Pc5 geomagnetic pulsations into two subgroups (Pc5A and Pc5B) based on spectral structure, J. Geomag. Geoelectr., 41, 813-834, 1989.

Lam, H. L. and G. Rostoker, The relationship of Pc5 micropulsation activity in the morning sector to the auroral westward electrojet, Planet. Space Sci., 26, 473-492, 1978.

Liu, J. Y., Y. N. Huang, and F. T. Berkey, The phase relationship between ULF geomagnetic pulsations and HF Doppler frequency shift oscillations on March 24, 1991, J. Geomag. Geoelectr., 45, 109-114, 1993. Reddy, C. A., S. Ravindran, K. S. Viswanathan, B. V. Krishna Murthy, D. R. K. Rao, and T. Araki, Observations of Pc5 micropulsation related electric field oscillations in the equatorial ionosphere, Ann. Geophys., 12, 565-573, 1994

Ruohoniemi, J. M., R. A. Greenwald, K. B. Baker, and J. C. Samson, Hf radar observations of Pc5 field line resonances in the midnight/early morning MLT sector, J. Geophys. Res., 96, 5697-15710, 1991.

Samson, J. C. and B. J. Harrold, Field line resonances associated with MHD waveguides in the magnetosphere, Geophys. Res. Lett., 19, 441444, 1992.

Samson, J. C., R. A. Greenwald, J. M. Ruohoniemi, T. J. Hughes, and D. D. Wallis, Magnetometer and radar observations of magnetohydromagnetic cavity modes in the Earth's magnetosphere, Can. J. Phys., 69, 929-937, 1991.

Sonnerup, B. O., Theory of the low-latitude boundary layers, J. Geophys. Res., 85, 2017-2026, 1980.

Southwood, D. J., Some features of field-line resonances in the magnetosphere, Planet. Space Sci., 22, 483-491, 1974.

Walker, A. D. M., J. M. Ruohoniemi, K. B. Baker, R. A. Greenwald, and J. C. Samson, Spatial and temporal behavior of ULF pulsations observed by the Goose bay HF radar, J. Geophys. Res., 97, 12187-12202, 1992.

Zhu, X. and M. G. Kivelson, Compressional ULF waves in the outer magnetosphere: 2. A case study of Pc5 type wave activity, J. Geophys. Res., 99, 241-252, 1994

Ziesolleck, C. W. S. and D. R. McDiarmid, Auroral latitude Pc5 field line resonances: Quantized frequencies, spatial characteristics and diurnal variation, J. Geophys. Res., 99, 5817-5830, 1994.

J. J. Schott (e-mail: schott@macareux.u-strasbg.fr), N. G. Kleimenova, J. Bitterly, and O. V. Kozyreva 